\title{
Plantes médicinales utilisées par les populations Bassa de la région de Douala au Cameroun
}

\author{
Siegfried Didier DIBONG ${ }^{1,2 *}$, Emmanuel MPONDO MPONDO ${ }^{2}$, Alfred NGOYE $^{3}$ and \\ Marie France KWIN ${ }^{1}$ \\ ${ }^{1}$ Département de Biologie des Organismes Végétaux, Faculté des Sciences, B.P. 24157, Université de Douala, \\ Cameroun. \\ ${ }^{2}$ Département des Sciences Pharmaceutiques, Faculté de Médecine et des Sciences Pharmaceutiques, B.P. \\ 2701, Université de Douala, Cameroun. \\ ${ }^{3}$ Institut de Recherche en Ecologie Tropicale, B.P. 13354, Libreville, Gabon. \\ *Auteur correspondant, E-mail: didierdibong@yahoo.fr
}

\section{RESUME}

Une enquête ethnobotanique portant sur un échantillon de 90 ménages a été conduite en juin 2010 dans trois villages de la région de Douala, au Cameroun, afin de recenser les plantes médicinales utilisées par les populations rurales. Elle montre que 48 espèces relevant de 44 genres et de 26 familles sont utilisées dans la pharmacopée traditionnelle. Les principales familles recensées sont les Fabaceae, les Euphorbiaceae et les Asteraceae, avec respectivement 6,5 et 4 espèces. Les fragments ou organes végétatifs collectés provenaient des jardins de case, vergers, jachères, lisières forestières ou plantations. Les espèces collectées représentent près de $94 \%$ de la flore. Seulement 3 espèces (environ 6\%) ont été échantillonnées en forêt. Les espèces exploitées renferment près de $42 \%$ d'herbacées et de $58 \%$ de ligneux. Les fragments ou organes les plus utilisés comprennent les feuilles, les fruits, les graines et les écorces.

() 2011 International Formulae Group. All rights reserved.

Mots clés: Pharmacopée traditionnelle, plante médicinale, population rurale, Douala, Cameroun.

\section{INTRODUCTION}

Les plantes médicinales constituent des ressources précieuses pour la grande majorité des populations rurales en Afrique dont plus de $80 \%$ se servent pour assurer leurs soins de santé (Jiofack et al., 2009, 2010 ; Betti et Mebere, 2011 ; Dibong et al., 2011 ; Ngono et al., 2011). En raison de l'enclavement des zones rurales, des infrastructures sanitaires inexistantes ou rudimentaires, du coût élevé des préparations pharmaceutiques, des faibles revenus, les plantes médicinales constituent un atout majeur pour les populations rurales. Dans la région de Douala, la majeure partie de la population utilise des plantes locales ou exotiques pour leurs soins de santé. Ces plantes médicinales ont fait l'objet d'un recensement exhaustif de leurs vertus curatives (avérées ou pas), et ont été identifiées par de nombreux auteurs (Adjanohoun et al., 1996; Dijk, 1999; Betti, 2002a, 2002b).

Le but de ce travail est de recenser les plantes médicinales exploitées par les populations rurales dans la région de Douala, au Cameroun, afin de mieux valoriser cette pharmacopée. 


\section{MATERIEL ET METHODES \\ Site de l'étude}

Douala, ville située au Sud-Ouest du Cameroun (03ㄴ $40^{\prime}-04^{\circ} 11^{\prime} \mathrm{N}, 09^{\circ} 16^{\prime}-09^{\circ} 52^{\prime}$ E, $13 \mathrm{~m}$ d'altitude) a un climat de type équatorial, caractérisé par deux saisons dont l'une pluvieuse et longue d'au moins 9 mois. Il est marqué par des précipitations abondantes (environ $4000 \mathrm{~mm}$ par an), et une température moyenne journalière élevée $\left(26,7{ }^{\circ} \mathrm{C}\right)$ variant faiblement sur toute l'année. Sur une période de 30 ans (19611990), la température maximale journalière atteint en moyenne $32,3{ }^{\circ} \mathrm{C}$ en février et 22,6 ${ }^{\circ} \mathrm{C}$ en juillet. L'humidité relative moyenne journalière est proche de la saturation sur toute l'année (Din et al., 2008).

\section{Enquête ethnobotanique}

Une enquête ethnobotanique sur les plantes médicinales utilisées dans la pharmacopée traditionnelle a été conduite en juin 2010 auprès d'une trentaine de ménages relevant de trois villages (Bomkoul, Massoumbou et Konbodol) dans la région de Douala, au Cameroun, situés respectivement à 17,21 et $27 \mathrm{~km}$ de ladite agglomération. Une quinzaine d'étudiants en Master I de Biologie des Organismes Végétaux de la Faculté des Sciences de l'Université de Douala, répartis en 3 groupes de 5 ont été mis à contribution pour ce travail. Chaque groupe comprenait au moins un étudiant issu de la tribu Bassa, afin de pouvoir communiquer aisément avec les ménages échantillonnés. Afin d'acquérir des informations fiables et authentiques sur les plantes médicinales et leurs vertus, les personnes interrogées étaient surtout des mères de familles relativement âgées. Les informations obtenues sur les pathologies traitées et leurs symptômes ont été recoupées auprès des médecins, infirmiers ou agents de santé locaux et à travers la littérature ethnobotanique (Adjanohoun et al., 1989). Dans l'optique d'une exploitation pratique des données collectées, celles-ci ont été mises en adéquation avec la classification des maladies proposée par l'OMS (OMS 1994, 1995, 1996, 2000; Adjanohoun et al., 1996). Les fiches d'enquête utilisées visaient à renseigner sur les fragments ou organes de plantes médicinales exploités, les techniques de collecte de ceux-ci, les méthodes de préparation, les maladies traitées et la posologie des médicaments administrés.

\section{Collecte des données}

Les échantillons des plantes ont été collectés à l'aide d'un sécateur et leur identification faite avec la collaboration des botanistes de la Faculté des Sciences de l'Université de Douala et de l'Herbier du Jardin Botanique de Limbé au Cameroun. Toutes les espèces déterminées ont été répertoriées et étiquetées.

\section{RESULTATS}

\section{Espèces recensées et origine}

Quarante huit (48) espèces de plantes médicinales, réparties en 44 genres et 26 familles ont été recensées dans les trois localités retenues : Bomkoul, Massoumbou et Konbodol (Tableau 1). Les trois principales familles recensées sont constituées des Fabaceae, des Euphorbiaceae et des Asteraceae représentées, respectivement, par 6, 5 et 4 espèces. Les fragments ou organes végétatifs ont été collectés dans des jardins de case, vergers, jachères, lisières forestières ou plantations. Les espèces collectées représentent près de $94 \%$ de la flore. Certaines d'entre elles sont devenues rares dans la région suite à des périodes successives de sécheresse intense. Très peu de fragments ou organes végétatifs, issus de 3 espèces (soit $6 \%$ de l'effectif total), ont été collectés en forêt. 
S. D. DIBONG et al. /Int. J. Biol. Chem. Sci. 5(3): 1105-1117, 2011

Tableau 1 : Liste des plantes médicinales utilisées en pharmacopée populaire à Bomkoul, Massoumbou et Konbodol.

\begin{tabular}{|c|c|c|c|c|c|}
\hline Famille & Espèces & Ligneux & herbacées & Nombre de genres & Nombre d'espèces \\
\hline Acanthaceae & Eremomastax speciosa (Hochst.) Cufod. & + & & 1 & 1 \\
\hline Anacardiaceae & Mangifera indica $\mathrm{L}$. & + & & 1 & 1 \\
\hline Annonaceae & Annona muricata $\mathrm{L}$. & + & & 1 & 1 \\
\hline Apocynaceae & $\begin{array}{l}\text { Alstonia boonei De Wild. } \\
\text { Voacanga africana Stapf }\end{array}$ & $\begin{array}{l}+ \\
+\end{array}$ & & 2 & 2 \\
\hline Arecaceae & $\begin{array}{l}\text { Cocos nucifera L. } \\
\text { Elaeis guineenis Jacq. }\end{array}$ & $\begin{array}{l}+ \\
+ \\
\end{array}$ & & 2 & 2 \\
\hline Asteraceae & 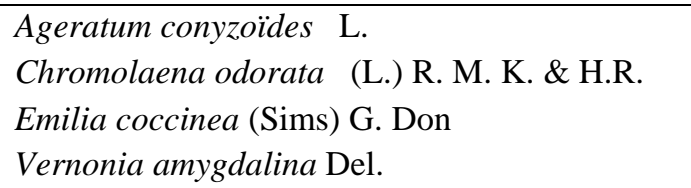 & $\begin{array}{l}+ \\
+\end{array}$ & $\begin{array}{l}+ \\
+\end{array}$ & 4 & 4 \\
\hline Burseraceae & Dacryodes edulis (G. Don.) H. J. Lam & + & & 1 & 1 \\
\hline Caparidaceae & Cleome ciliata $\mathrm{L}$. & & + & 1 & 1 \\
\hline Caricaceae & Carica papaya $\mathrm{L}$. & + & & 1 & 1 \\
\hline Clusiaceae & Garcinia cola Heck. & + & & 1 & 1 \\
\hline Costaceae & Costus afer Ker-Gawl. & & + & 1 & 1 \\
\hline Dioscoreaceae & Dioscorea sp. & & + & 1 & 1 \\
\hline & $\begin{array}{l}\text { Alchornea cordifolia (Schum. \& Thonn.) Müll. } \\
\text { Arg }\end{array}$ & + & & 4 & 4 \\
\hline Euphorbiaceae & $\begin{array}{l}\text { Manihiot esculenta Crantz } \\
\text { Manniophyton fulvum Müll. Arg. } \\
\text { Ricinodendron heudolotii (Baill.) Pierre ex Pax }\end{array}$ & $\begin{array}{l}+ \\
+ \\
+\end{array}$ & & & \\
\hline Fabaceae & $\begin{array}{l}\text { Arachis hypogaea } \mathrm{L} . \\
\text { Cassia alata } \mathrm{L} . \\
\text { Cassia occidentalis } \mathrm{L} . \\
\text { Mimosa pudica } \mathrm{L} .\end{array}$ & $\begin{array}{l}+ \\
+\end{array}$ & + & 5 & 6 \\
\hline
\end{tabular}


S. D. DIBONG et al. /Int. J. Biol. Chem. Sci. 5(3): 1105-1117, 2011

Phaseolus vulgaris L.

Scorodophleus zenkeri Harms

\begin{tabular}{|c|c|c|c|c|c|}
\hline & Scorodophleus zenkeri Harms & + & & & \\
\hline Gnetaceae & Gnetum africanum Welw. & & + & 1 & 1 \\
\hline Irvingiaceae & $\begin{array}{l}\text { Irvingia gabonensis (Aubey-Lec ex O'Rorke) } \\
\text { Mill. }\end{array}$ & + & & 1 & 1 \\
\hline Lamiaceae & Ocimum basilicum $\mathrm{L}$. & + & & 1 & 1 \\
\hline Lauraceae & Persea americana Mill. & + & & 1 & 1 \\
\hline \multirow{3}{*}{ Liliaceae } & Allium сера L. & & + & \multirow{3}{*}{2} & \multirow{3}{*}{3} \\
\hline & Alluim sativum $\mathrm{L}$. & & + & & \\
\hline & Aloe vera $\mathrm{L}$. & & + & & \\
\hline Loranthaceae & Phragmanthera capitata (Spreng.) S. Balle & + & & 1 & 1 \\
\hline \multirow{2}{*}{ Musaceae } & Musa paradisiaca $\mathrm{L}$. & & + & \multirow{2}{*}{1} & \multirow{2}{*}{2} \\
\hline & Musa sapientum L. & & + & & \\
\hline \multirow{2}{*}{ Myrtaceae } & Eucaluptus sailgna Smith. & + & & \multirow{2}{*}{2} & \multirow{2}{*}{2} \\
\hline & Psidium guajava L. & + & & & \\
\hline Passifloraceae & Passiflora sp. & & + & 1 & 1 \\
\hline \multirow{3}{*}{ Poaceae } & Cympobogon citratus Stapf & & + & \multirow{3}{*}{3} & \multirow{3}{*}{3} \\
\hline & Saccharum officinarum L. & & + & & \\
\hline & Zea mays L. & & + & & \\
\hline \multirow{2}{*}{ Rutaceae } & Citrus limon (L.) Burm. f. & + & & \multirow{2}{*}{1} & \multirow{2}{*}{2} \\
\hline & Citrus aurantifolia (Christm.) Swingle & + & & & \\
\hline \multirow{2}{*}{ Solanaceae } & Capsicum frutescens $\mathrm{L}$. & + & & \multirow{2}{*}{2} & \multirow{2}{*}{2} \\
\hline & Solanum tuberosum L. & & + & & \\
\hline Zingiberaeae & Zingiber officinale Rosc. & & + & 1 & 1 \\
\hline
\end{tabular}


S. D. DIBONG et al. /Int. J. Biol. Chem. Sci. 5(3): 1105-1117, 2011

Tableau 2: Liste des plantes médicinales utilisées en pharmacopée populaire au village Bomkoul (P.K. 17).

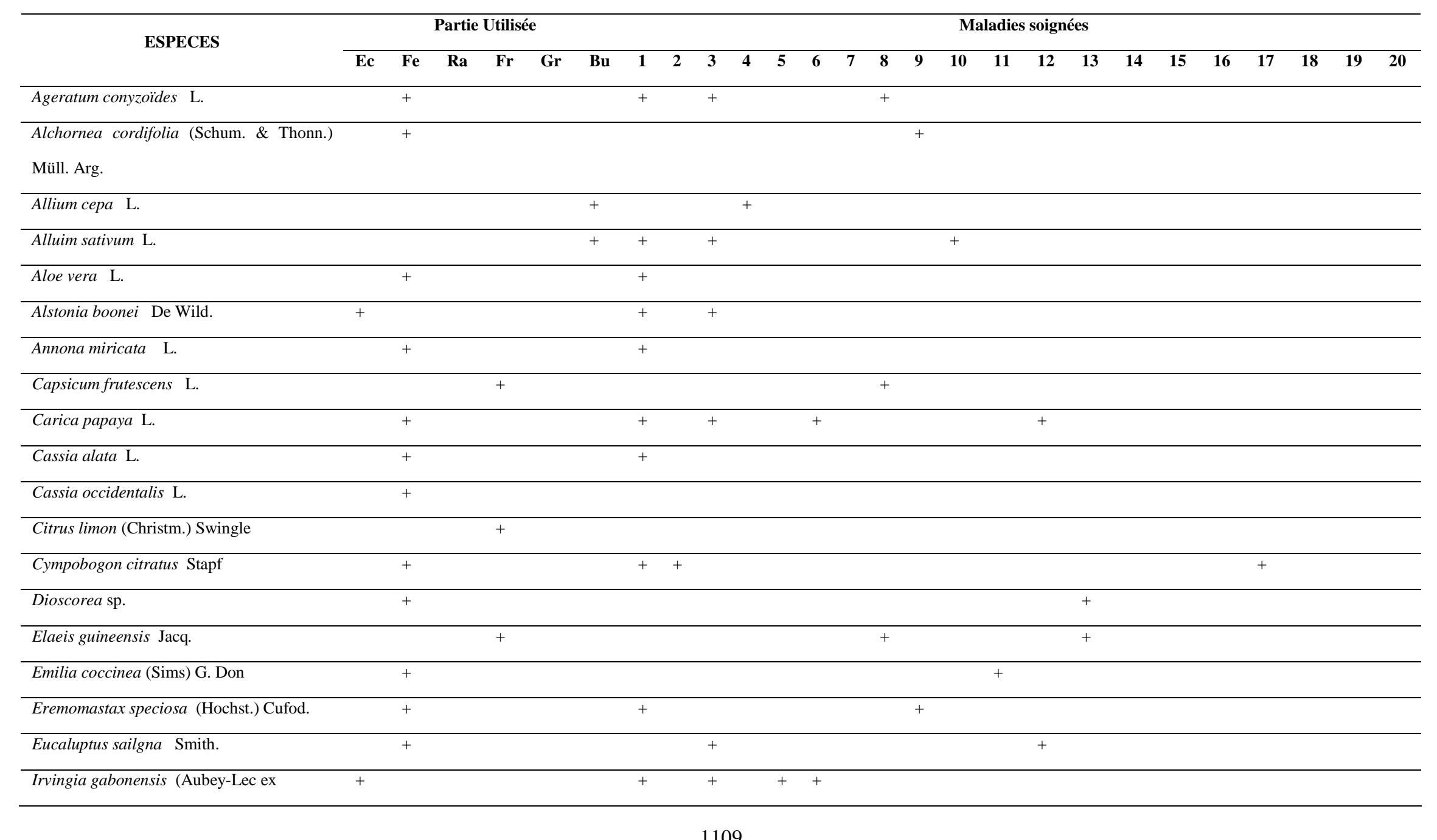


S. D. DIBONG et al. /Int. J. Biol. Chem. Sci. 5(3): 1105-1117, 2011

O'Rorke) Mill.

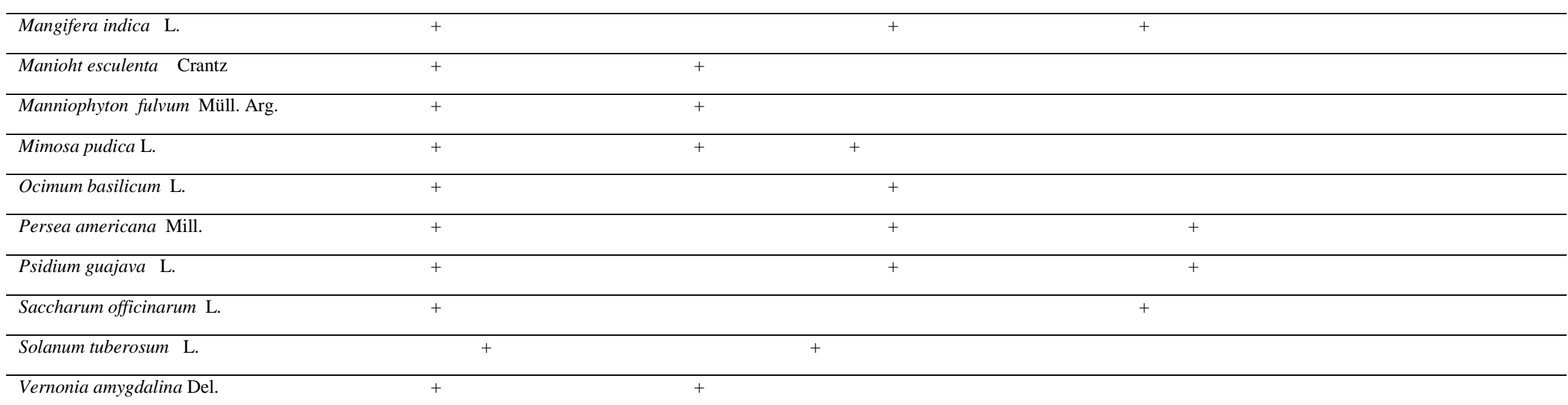

Organe végétal : Ec : écorce de tige ; Fe : Feuille ; Ra : racine ; gr : graine ; Fr : fruit ; Bu : Bulbe.

Maladies soignées : 1- Paludisme ; 2- Grippe ; 3- Typhoïde ; 4- Mal d'estomac ; 5- Mal de dent ; 6- Mal de ventre ; 7- Mal de tête ; 8- Mal de rein ; 9- Anémie ; 10 - Démangeaison ; 11-Tension ; 12 Jaunisse ; 13-Vers intestinaux/14- Diabète ; 15- Mal de dos ; 16- Diarrhée ; 17- Toux ; 18- Rhume ; 19- Asthme ; 20-Mal de nerf.

Tableau 3 : Liste des plantes médicinales utilisées en pharmacopée populaire au village de Massoumbou (P.K. 21).

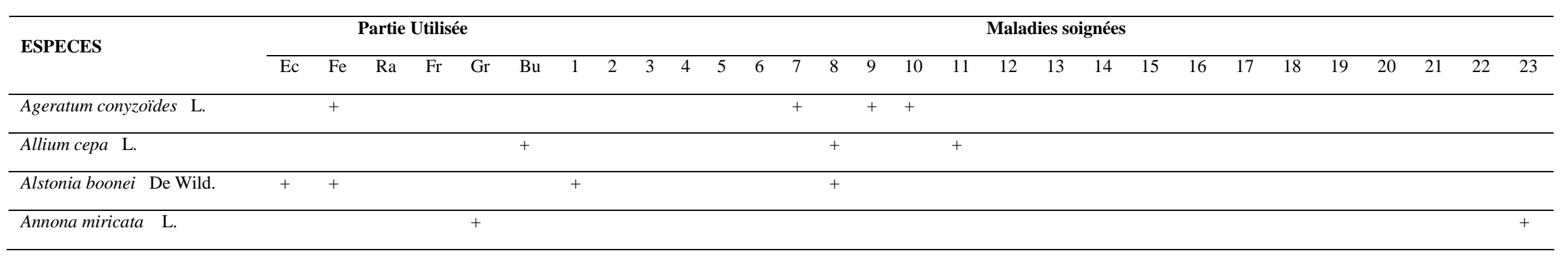


S. D. DIBONG et al. /Int. J. Biol. Chem. Sci. 5(3): 1105-1117, 2011

Chromolaena odorata (L.) + + +

\section{R.M.K. \& H.R.}

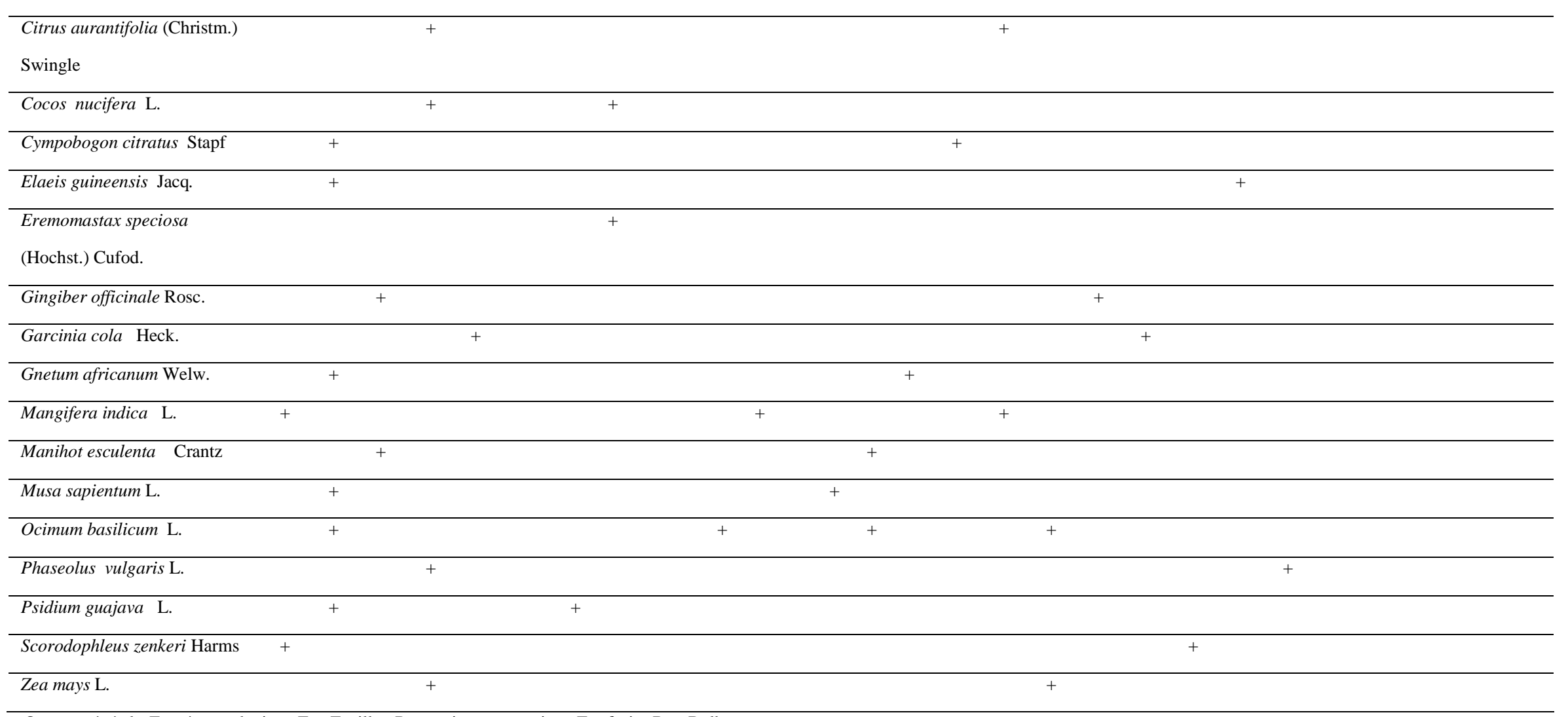

Organe végétal : Ec : écorce de tige ; Fe : Feuille ; Ra : racine ; gr : graine ; Fr : fruit ; Bu : Bulbe.

Maladies soignées : 1- Paludisme ; 2-Rouge fesse ; 3- Hernie ; 4-Fontanelle ; 5-Mal de ventre ; 6-Mal de dent ; 7- Plaie ; 8-Rate ; 9-Mal d'estomac ; 10-Panaris ; 11-Tension ; 12-Cholera ; 13-Rhumatisme ; 14-Toux ; 15- Indigestion ; 16-Chlamydia ; 17- Chaude pisse ; 18- Tremblote ; 19- Oedèmes 20- Mal de nerf ; 21-Vers ; 22- Diarrhée ; 23-Cancers. 
S. D. DIBONG et al. /Int. J. Biol. Chem. Sci. 5(3): 1105-1117, 2011

Tableau 4 : Liste des plantes médicinales utilisées en pharmacopée populaire au village de Konbodol (P.K. 27).

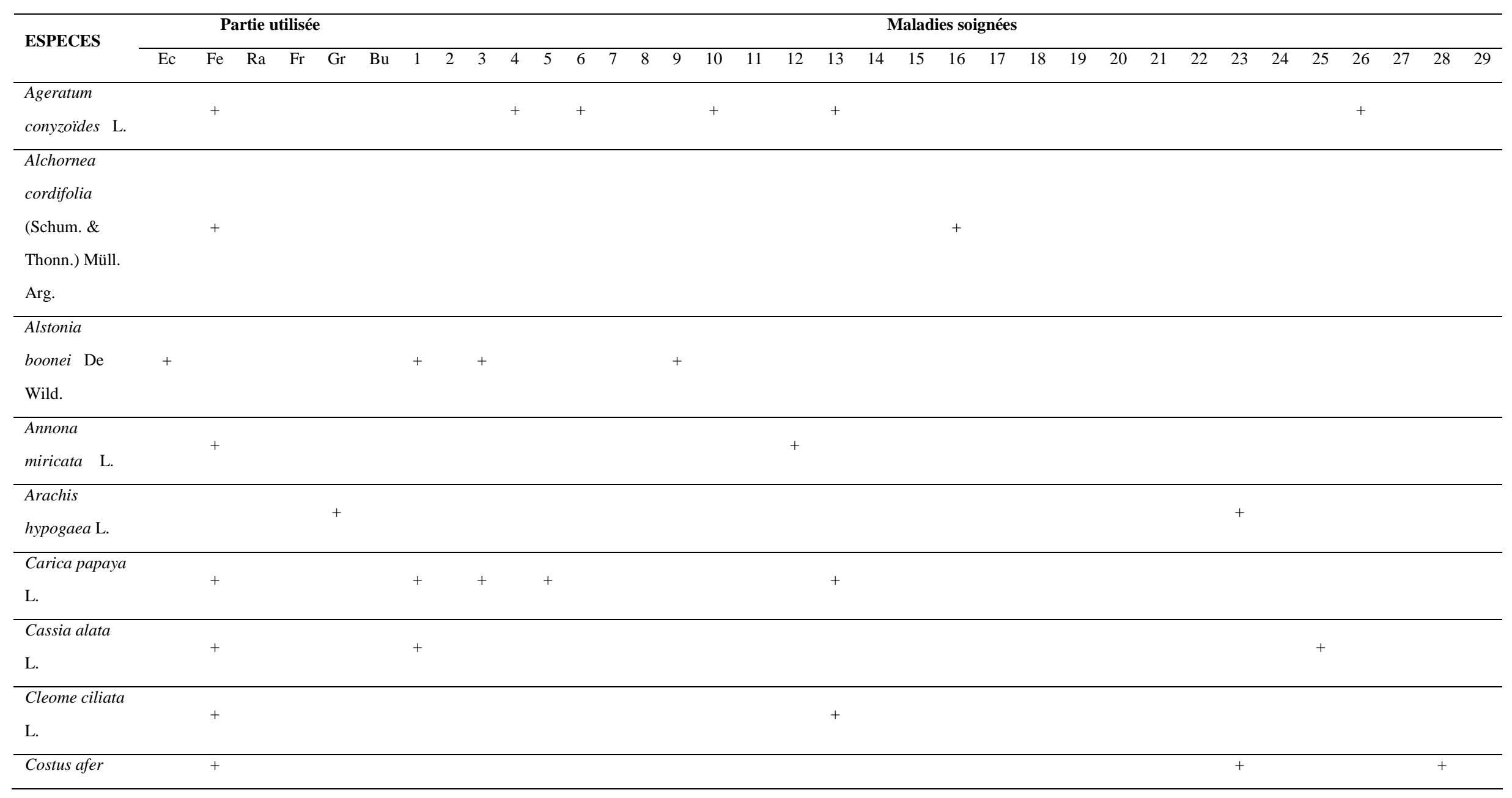


S. D. DIBONG et al. /Int. J. Biol. Chem. Sci. 5(3): 1105-1117, 2011

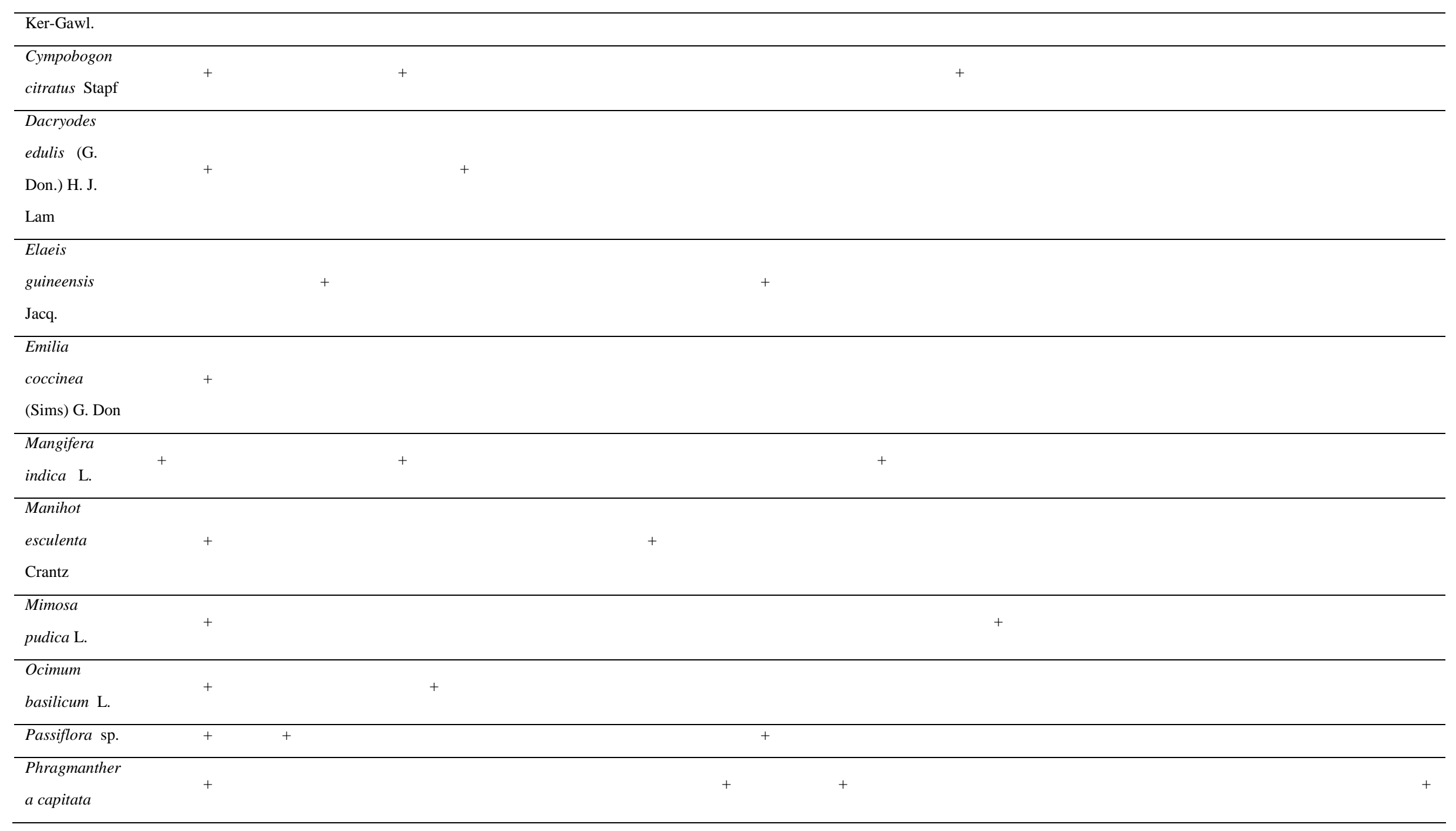


S. D. DIBONG et al. /Int. J. Biol. Chem. Sci. 5(3): 1105-1117, 2011

\section{(Spreng.) S.}

\section{Balle}

Psidium

guajava L.

Ricinodendron

heudolotii

(Baill.) Pierre

ex Pax

Vernonia

amygdalina

$+$

$++$

Voacanga

africana Stapf

Organe végétal : Ec : écorce de tige ; Fe : Feuille ; Ra : racine ; gr : graine ; Fr : fruit ; Bu : Bulbe.

Maladies soignées : 1- Paludisme ; 2-Grippe ; 3-Typhoïde ; 4- Mal d'estomac ; 5- Carie dentaire ; 6- Mal de ventre ; 7- Maux de tête ; 8- Rate ; 9- Anémie ; 10- Morsure de serpent ; 11- Tension ; 12-

Jaunisse ; 13- Vers intestinaux ; 14- Diabète ; 15- Hémorroïde ; 16- Diarrhée ; 17- Toux ; 18- Panaris ; 19- Bronchite ; 20- Mal de nerfs ; 21- Rhumatisme ; 22- Constipation ; 23- Rougeole ; 24- indigestion ;

25- Dartre ; 26- Blessure ; 27- Mal des yeux ; 28-Variole ; 29- Cancer 


\section{Espèces exploitées et fragments ou organes prélevés}

Les espèces de plantes médicinales exploitées sont de nature herbacée $(41,7 \%)$ ou ligneuse $(58,3 \%)$. Ce sont des espèces ubiquistes, eu égard à la variabilité importante de leur habitat. Elles sont de petite taille et présentent une maturité précoce. Elles ont une descendance nombreuse et se distinguent par des graines à longue période de dormance qui permet d'expliquer en partie leur nature ubiquiste (Pianka, 1970).

Les fragments ou organes végétatifs les plus exploités comprennent les feuilles, les fruits, les graines et les écorces (Tableaux 2, 3 et 4).

\section{Pérennité des plantes médicinales utilisées}

Afin de faire face à la pénurie de certaines plantes médicinales, les ménages enquêtés ont proposé diverses techniques telles que celles basées sur des prélèvements moins traumatisants pour la plante, la culture d'espèces dans des jardins de case ou dans des jardins dédiés aux plantes médicinales.

\section{DISCUSSION}

Les populations rurales utilisent de nombreuses espèces végétales comme plantes médicinales dont la plupart sont disponibles dans leur environnement immédiat, ce qui limite la pression humaine sur l'environnement (Betti et Mebere, 2011; Dibong et al., 2011 ; Ngono et al., 2011).

Parmi les plantes utilisées dans la pharmacopée traditionnelle, nombreuses sont celles qui ont été insuffisamment étudiées scientifiquement (Adjanohoun, 2000). Des efforts devraient être faits pour améliorer cette situation afin de mieux valoriser la pharmacopée traditionnelle.

L'importance des feuilles, des fruits, des graines, ainsi que des écorces parmi les fragments ou organes végétatifs collectés peut être attribuée à leur facilité de prélèvement par les populations locales (Betti, 2002a). Pour les espèces herbacées, la technique de collecte consiste en l'arrachage des jeunes tiges ou à la coupe des tiges en morceaux. Le mode d'exploitation des écorces et des racines pratiqué actuellement ne garantit pas toujours la pérennité de l'espèce. Cunningham (1988, 1990) considère les arbres comme les types morphologiques les plus vulnérables à ce mode d'exploitation. En effet, l'écorçage systématique des arbres à cernes pratiqué par les populations locales provoque leur dépérissement à plus ou moins long terme. Les plantes vivaces à bulbes ou tubercules représentent, selon Cunningham (1991), une autre catégorie d'espèces menacées par une exploitation abusive de leurs parties souterraines.

La pharmacopée traditionnelle pratiquée dans les localités visitées, tout comme dans le Dja (Betti, 2002a) ou en Afrique centrale (Diafouta, 1997) ne dispose pas de méthodes aseptiques pour conserver les médicaments en milieux aqueux (macérations, décoctions). L'utilisation est immédiate dans le cas des espèces herbacées, mais dans celui des écorces d'espèces ligneuses, la conservation n'excède pas trois jours. Betti (2002a) et Ngono et al. (2011) observent que la conservation peut durer jusqu'à cinq voire sept jours dans la région du Dja. Au terme de ce délai, le remède devient impropre à la consommation, inefficace et toxique avec la formation d'une mousse blanche importante à la surface du liquide. Les formes aqueuses sont dominantes dans ces villages en matière de pharmacopée traditionnelle (Betti, 2002a). Les formes médicamenteuses conservées par séchage à l'ombre ou au soleil à l'état de poudre, cendre ou pommade sont plutôt rares. La faible importance des arbres issus de la forêt secondaire ou primaire dans la pharmacopée s'explique par leur éloignement des habitations et par l'environnement immédiat qui apporte déjà les solutions aux préoccupations de santé des populations rurales.

La création d'une base de données sur les plantes médicinales utilisées par les populations locales s'avère nécessaire. Pour ce faire, les associations de tradi-praticiens devraient travailler en collaboration étroite avec des pépiniéristes pour un meilleur 
développement des plantes médicinales. La création de jardins botaniques ou de jardins dédiés aux plantes médicinales devrait être initiée par les ONGs et des associations œuvrant pour la sauvegarde de la biodiversité végétale.

\section{Conclusion}

L'enquête ethnobotanique a permis de dénombrer 48 espèces de plantes médicinales, réparties en 44 genres et 26 familles dans la région de Douala au Cameroun. Les trois principales familles recensées sont constituées des Fabaceae, des Euphorbiaceae et des Asteraceae représentées, respectivement, par 6,5 et 4 espèces. L'exploitation de ces plantes médicinales par les populations rurales a un faible impact négatif sur l'environnement. Elle mériterait donc d'être encouragée et valorisée. En effet, l'intensité et les fréquences de prélèvement des fragments ou organes végétatifs effectués dans ces localités, permettent d'assurer le renouvellement de la ressource à des fins thérapeutiques.

\section{REMERCIEMENTS}

Nous remercions sincèrement les étudiants en Master I de Biologie des Organismes Végétaux de la Faculté des Sciences de l'Université de Douala, promotion 2009/2010.

\section{REFERENCES}

Adjanohoun E, Cusset G, Issa LO, Keita A, Lebras M, Lejoly J, Waechter P. 1989. Banque de données de médecine traditionnelle et de pharmacopée (pharmel). Notice pour la récolte et l'entrée des données, ACCT, Paris.

Adjanohoun E, Aboubakar N, Dramane K, Ebot ME, Ekpere JA, Enow-Orock EG, Focho D, Gbile ZO, Kamanyi A, Kamsu Kom J, Keita A, Mbenkum T, Mbi CN, Mbiele AL, Mbome IL, Mubiru NK, Nancy WL, Nkongmeneck B, Satabie B, Sofowora A, Tamze V, Wirmum CK. 1996. Contribution to ethnobotanical and floristical studies in Cameroon. CSTR/OUA.
Adjanohoun E. 2000. La biodiversité face au développement des industries pharmaceutiques africaines. In Réseau des «Espèces Ligneuses Médicinales», Eyog Matig O, Adjanohoun E, de Souza S, Sinsin B (eds). Compte rendu de la première réunion du réseau tenue 15-17 décembre 1999 à la station IITA Cotonou, Bénin ; 88-103.

Betti JL. 2002a. Usages traditionnels des plantes médicinales et traitement des maux de dos dans la réserve de biosphère $\mathrm{du}$ Dja/Cameroun. In History of Health and Diseases: Living and Curing Old Age in The World, Gueri $\mathrm{A}$, Consiglière $\mathrm{S}$ (eds). Genoa/Italy; 117-154.

Betti JL. 2002b. Medicinal plants sold in Yaounde markets, Cameroon. African Study Monographs, 23(3): 47-64.

Cunningham AB. 1988. An investigation of the herbal medicine trade in Natal/Kwazulu. Investigational Report 29. Insttiute of Natural Ressources, University of Natal.

Betti JL, Mebere Yemefa SR. 2011. Contribution à la connaissance des produits forestiers non ligneux du parc de Kalamaloué, Extrême nord Cameroun: les plantes alimentaires. International Journal of Biological and Chemical Sciences, 5(1): 291-303.

Cunningham AB. 1990. Man and medicines: the exploitation and conservation of traditional zulu medicinal plants. In Proceedings of the XIIth AETFAT Congress, Ihlenfeldt HD, Baijnath $\mathrm{H}$, Cheep M, Hepper FN, Lejoly J, Lucas GL, Malaisse FP, Peters CR \& Wessels DCJ (ed). Mitteilungen aus dem Institut fur Allgemeine Botanik 23: Hambourg; 979-990.

Cunningham AB. 1991. Development of a conservation policy on commercially exploited medicinal plants: a case study from Southern Africa. In Conservation of Medicinal Plants, Akarele O, Heywood $\mathrm{V}$, Synge $\mathrm{H}$ (eds). Cambridge Univ. Press: Cambridge. 
Diafouka A. 1997. Analyse des usages des plantes médicinales dans quatre régions du Congo-Brazzaville. Thèse Doc., Univ. Libre de Bruxelles, p. 434.

Dibong SD, Mpondo Mpondo E, Ngoye A, Betti JL, 2011. Ethnobotanique et phtytomédecine des plantes médicinales vendues sur les marchés de Douala, Cameroun. Journal of Applied Biosciences, 37: 2496-2507.

Dijk JFW. 1999. Non-timber forest products in the Bipindi-Akom II region, Cameroon. A socio-economic and ecological assessment; The Tropenbos Cameroon Program.

Din N, Priso RJ, Dibong SD, Amougou A. 2008. Logging activities in mangrove forests: a case study of Douala Cameroon. African Journal of Environmental Science and Technology, 2(2): 22-30.

Jiofack T, Ayissi I, Fokunang C, Guedje N, Kemeuze V. 2009. Etnobotany and phytomedicine of the upper Nyong Valley forest in Cameroon. African Journal of Pharmacy and Pharmacology, 3(4): 144-150.

Jiofack T, Fokunang C, Guedje N, Kemeuze $\mathrm{V}$, Fongnzossie E, Nkongmeneck BA, Mapongmetsem PM, Tsabang N. 2010. Ethnobotanical uses of medicinals plants of two ethnoecological regions of Cameroon. International Journal of Medicine and Medical Sciences, 2(3): 60-79.
Ngono Ngane RA, Koanga Mogtomo ML, Tchinda Tabou A, Magnifouet Nana H, Motso Chieffo PR, Mballa Bounou Z, Ebelle Etame RM, Ndifor F, Biyiti L, Amvam Zollo PH. 2011. Ethnobotanical survey of some camerounian plants used for treatment of viral diseases. African Journal of Plant Science, 5(1): 15-21.

Organisation Mondiale de la Santé (OMS). 1994. Classification Statistique Internationale des Maladies et des Problèmes de Santé Connexes (10ème révision, vol. 1). OMS : Genève.

OMS. 1995. Classification Statistique Internationale des Maladies et des Problèmes de Santé Connexes (10ème révision, vol. 2). OMS : Genève.

OMS. 1996. Classification Statistique Internationale des Maladies et des Problèmes de Santé Connexes (10ème révision, vol. 3). OMS : Genève.

OMS. 2000. Stratégie de l'OMS pour la médecine traditionnelle pour 20022005, Genève, 78p.

Pianka ER. 1970. On r- and k- selection. Am Nat., 104: 592-597. 